\title{
Lymph node trafficking and antigen presentation by endobronchial eosinophils
}

\author{
Huan-Zhong Shi, ${ }^{1}$ Alison Humbles, ${ }^{2}$ Craig Gerard, ${ }^{2}$ Zhuang Jin, ${ }^{1}$ and Peter F. Weller ${ }^{1}$ \\ ${ }^{1}$ Department of Medicine, Harvard Thorndike Laboratories, Charles A. Dana Research Institute, \\ Beth Israel Deaconess Medical Center, Harvard Medical School, Boston, Massachusetts 02215, USA \\ ${ }^{2}$ Department of Pediatrics, Ina Sue Perlmutter Laboratories, Children's Hospital Medical Center, \\ Harvard Medical School, Boston, Massachusetts, 02115 USA \\ Address correspondence to: Peter F. Weller, Beth Israel Deaconess Medical Center, DA-617, 330 Brookline Avenue, \\ Boston, Massachusetts 02215, USA. Phone: (617) 667-3307; Fax: (617) 277-6061; E-mail: pweller@caregroup.harvard.edu.
}

Received for publication November 19, 1999, and accepted in revised form February 15, 2000.

Because eosinophils recruited into the airways in allergic diseases are exposed to inhaled allergens, we evaluated whether eosinophils within the endobronchial lumen can function in vivo as antigen-presenting cells for inhaled antigens. We recovered eosinophils from the airways after aerosol antigen challenge in sensitized mice or from the peritoneal cavities of IL-5 transgenic mice and fluorescently labeled these cells ex vivo. These labeled cells, instilled intratracheally into normal mice, migrated into draining paratracheal lymph nodes and localized to $T$ cell-rich paracortical areas. The homing of airway eosinophils to lymph nodes was not governed by eotaxin, because $C C R 3^{-/-}$and $C C R 3^{+/+}$eosinophils migrated identically. Airway eosinophils, recovered after inhalational antigen challenge in sensitized mice, expressed MHC class II and costimulatory CD80 and CD86 proteins and functioned in vitro as CD80- and CD86-dependent, antigen-specific, antigen-presenting cells. Moreover, when instilled into the airways of antigen-sensitized recipient mice, airway eosinophils recovered after inhalational antigen challenge stimulated antigen-specific $\mathrm{CD}^{+} \mathrm{T}$ cell proliferation within paratracheal lymph nodes. Thus, eosinophils within the lumina of airways can process inhaled antigens, traffic to regional lymph nodes, and function in vivo as antigen-presenting cells to stimulate responses of $\mathrm{CD} 4{ }^{+} \mathrm{T}$ cells.

J. Clin. Invest. 105:945-953 (2000).

\section{Introduction}

In allergic diseases involving the lungs (asthma) and upper airways (allergic rhinitis), inhalational exposures to allergens lead to the recruitment of eosinophils into involved tissues and the appearance of intraluminal eosinophils in respiratory secretions. Many studies have delineated roles for recruited eosinophils and mechanisms contributing to their recruitment. In the tissues, eosinophils can have effector functions in promoting the pathogenesis of these diseases. Eosinophils release lipid mediators and contain distinct cationic granule proteins that may cause dysfunction and destruction of other cells (1). In rodent models and humans, allergeninduced recruitment of eosinophils into lung tissues has correlated with $\mathrm{CD}^{+} \mathrm{T}$ cells and cytokines (e.g., IL5 and GM-CSF) released by these $\mathrm{T}$ cells $(2-4)$. Thus, eosinophil accumulation and enhanced effector functions at tissue sites of allergic reactions may be related to lymphocyte activation, especially by Th2-like lymphocytes elaborating cytokines that enhance the effector responses of eosinophils.

In addition to the conventional effector functions of eosinophils based on their release of lipid mediators or cationic granule proteins, observations suggest that eosinophils may have functional roles in interactions with $\mathrm{T}$ and $\mathrm{B}$ lymphocytes. For potential interactions with lymphocytes, human eosinophils express relevant membrane proteins, including CD40 (5), CD28, and
CD86 (6), and may be induced to express MHC class II antigens (7). In addition, eosinophils are sources of cytokines capable of stimulating lymphocytes, including IL-2, IL-12, IL-4, and IL-10 (8). In vitro, human and murine eosinophils can function as antigen-presenting cells (APCs) $(9,10)$, but as yet there is no information concerning functional interactions between eosinophils and lymphocytes in vivo.

Although the presence of eosinophils within airway secretions is characteristic of allergic diseases of the airways, including asthma and rhinitis $(11,12)$, functional roles for eosinophils within the lumina of the airways are not known. Such intraluminal eosinophils, either naturally present in the sputum of those with asthma (13) or elicited after segmental antigen (14) or inhalational (15) challenges in allergic subjects, express HLADR. Thus, allergen challenge elicits not only eosinophil influx into the airways, but also induces recruited eosinophils to express HLA-DR not found on otherwise phenotypically activated blood eosinophils $(14,15)$. Airway epithelial cells may produce eosinophil chemoattractants, such as IL-16 (16), RANTES (17), and eotaxin (18); RANTES has a polarized expression specifically on the luminal surface of the epithelium (19).

Does the recruitment of eosinophils into the secretions of the airways reflect solely the agonal response of these leukocytes or might intraluminal eosinophils have functional responses within the airways where 
they would encounter inhaled allergens? We have examined the trafficking of fluorescently labeled eosinophils from within the trachea into regional lymph nodes and evaluated whether eosinophils encountering antigen within the airways have the capabilities to process and present such antigens to lymphocytes in peritracheal lymph nodes. We show that eosinophils within the airways are capable of processing inhaled antigens and migrating to lymphoid tissue to effectively present these antigens to $\mathrm{CD}^{+} \mathrm{T}$ cells.

\section{Methods}

Animals. BALB/c and $\mathrm{C} 3 \mathrm{H} / \mathrm{HeN}$ mice were from Charles River Laboratories (Wilmington Massachusetts, USA). Mice with a genetic deletion of the CCR3 chemokine receptor $\left(C C R 3^{-/}\right)$on a BALB/c background and their wild-type littermates $\left(C C R 3^{+/+}\right)$were developed as described (A.A. Humbles et al., manuscript submitted). Breeding pairs of IL-5 transgenic $\mathrm{C} 3 \mathrm{H} / \mathrm{HeN}$ mice (20) were kindly provided by Akira Tominaga (Kochi Medical School, Kochi, Japan).

Preparation of eosinophils. BALB/c, CCR3 $3^{-/}$, or $\mathrm{CCR}^{+/+}$ wild-type littermate mice were sensitized intraperitoneally 3 times with $10 \mu \mathrm{g}$ of either OVA, BSA, or human gamma globulin (HGG; Sigma Chemical Co., St. Louis, Missouri, USA) plus $1 \mathrm{mg} \mathrm{Al}(\mathrm{OH})_{3}$ in $0.2 \mathrm{~mL}$ PBS on day 1,7 , and 14 . On day 21, 22, 23, and 24, sensitized mice underwent inhalational challenges with homologous antigen by daily exposures to aerosolized antigen (5\% in PBS) delivered for 30 minutes by a DeVilbiss 646 nebulizer (DeVilbiss, Somerset, Pennsylvania, USA). Twenty-four hours after the final challenge, mice were given a lethal dose of pentobarbital $(60 \mathrm{mg} / \mathrm{kg}$ intraperitoneally), and the lungs were lavaged 6 times with 0.8 - $\mathrm{mL}$ aliquots of PBS containing $4 \mathrm{mM}$ EDTA and $2 \%$ mouse serum (PBS/serum) to obtain bronchoalveolar lavage (BAL) for eosinophil purification. In specified studies, eosinophils were isolated from peritoneal lavages of IL-5 transgenic mice that were injected intraperitoneally with $0.5 \mathrm{mg} / \mathrm{kg} \mathrm{CdCl} 2$ in $0.2 \mathrm{~mL}$ PBS 48 hours before five 5 - $\mathrm{mL}$ intraperitoneal lavages with PBS/serum. Eosinophils were purified on discontinuous Percoll density gradients followed by immunomagnetic purification. Four densities (1.085, 1.080, 1.075 , and $1.070 \mathrm{gm} / \mathrm{mL}$ ) of Percoll (adjusted to isotonicity by adding 1 part $10 \times$ PBS to 9 parts Percoll) were prepared with PBS. Five milliliters of each decreasing density were sequentially layered in $50-\mathrm{mL}$ plastic tubes, and cells in $5 \mathrm{~mL}$ PBS/serum were layered on top. After centrifugation (25 minutes, $1,500 \mathrm{~g}$, room temperature), eosinophils were between the 1.075 and $1.070 \mathrm{~g} / \mathrm{mL}$ layers, with most macrophages on top of the gradients. Collected eosinophils were washed in $\mathrm{PBS} /$ serum, and, after 1 hour of culture on plastic Petri dishes in RPMI-1640 supplemented with penicillin $(100 \mathrm{U} / \mathrm{mL})$, streptomycin $(100 \mu \mathrm{g} / \mathrm{mL})$, L-glutamine (2 mM), HEPES (10 $\mathrm{mM})$, and $5 \%$ mouse serum to eliminate remaining adherent macrophages, were further purified by negative immunomagnetic selection
(MACS; Miltenyi Biotec, Auburn, California, USA). To remove contaminating lymphocytes, anti-CD90- and anti-CD19-coated micromagnetic beads were added. By negative selection, more than $99.5 \%$ pure eosinophils were obtained as assessed by microscopy and flow cytometry. Viability by trypan blue exclusion of purified eosinophils was more than $97 \%$.

Trafficking offluorescently labeled eosinophils in vivo. Purified eosinophils were labeled with the red fluorescent dye, 1,1'-dihexadecyl-3,3,3',3'-tetramethylindocarbocyanin perchlorate $\left[\mathrm{DiIC}_{16}(3)\right]$, according the manufacturer's protocol (Molecular Probes, Eugene, Oregon, USA), and after labeling were more than $96 \%$ viable by trypan blue exclusion. The tracheas of mice anesthetized with Avertin $(250 \mu \mathrm{g} / \mathrm{kg})$ were surgically exposed. DiIC $_{16}(3)$-labeled eosinophils $\left(5 \times 10^{5}\right.$ in $50 \mu \mathrm{L}$ PBS) were instilled slowly into the trachea with a 25gauge needle. Mice were sacrificed at different times, and paratracheal, cervical, axillary, brachial, inguinal, and popliteal lymph nodes, as well as lungs, livers, and spleens, were harvested and fixed in $2 \%$ paraformaldehyde for 1 hour at $4^{\circ} \mathrm{C}$ and then infused with $30 \%$ sucrose solution overnight. Tissues were frozen with liquid nitrogen, embedded in OCT compound (Fisher
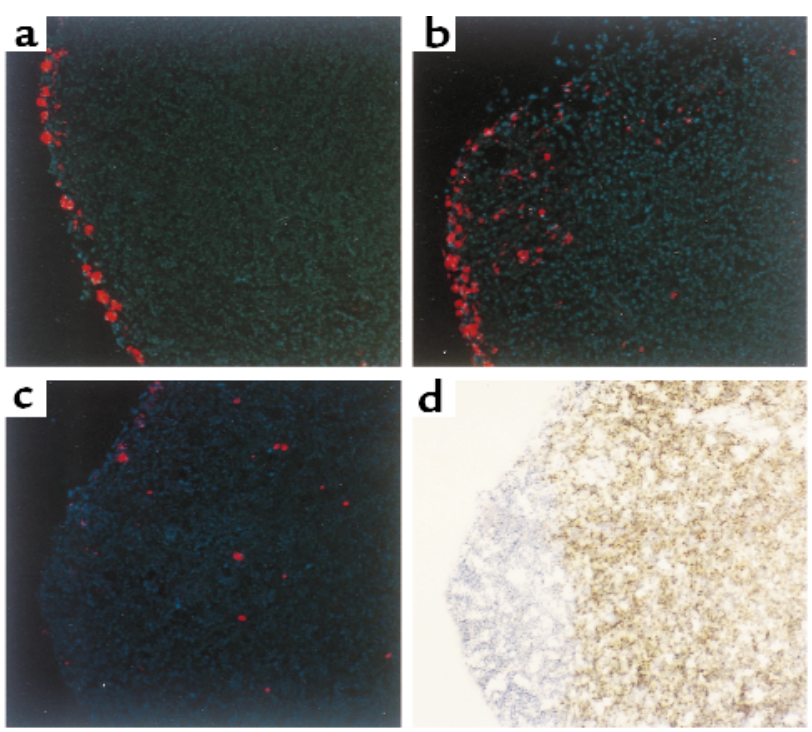

\section{Figure 1}

Eosinophil migration from the airways into paratracheal lymph nodes. Eosinophils $\left(5 \times 10^{5}\right)$ from the airways of antigen-sensitized and aerosol-challenged mice and labeled with $\operatorname{DilC}_{16}(3)$ (red), were instilled into the tracheas of recipient mice. Paratracheal lymph nodes were harvested at the indicated times after eosinophil instillation. Cryosectioned lymph nodes were stained with HOECHST 33342 (blue) to highlight nuclei and examined by fluorescence microscopy. T cell-rich regions were identified by immunoperoxidase staining with anti-CD3 mAb (brown). Labeled eosinophils were visible in the marginal sinus and the subcapsular region at 8 hours after instillation (a) and started to stream through the subcapsular sinus moving into the paracortical area at 16 hours (b). By 24 hours labeled eosinophils were predominantly located in the T-cell area, whereas few eosinophils were located in B-cell areas and in the subcapsular sinus (c, d). $\mathbf{c}$ and $\mathbf{d}$ are images of the same section for comparison. (Originally $\times 250$.) 
Scientific, Medford, Massachusetts, USA) and stored at $-70^{\circ} \mathrm{C}$. Frozen tissues were cryosectioned $(10-\mu \mathrm{m}$ thick sections), collected onto Superfrost slides (Fisher), and incubated with HOECHST 33342 (Sigma) to stain cell nuclei. Slides were examined by fluorescence microscopy, and images were photographed by double exposures with filters appropriate for $\mathrm{DiIC}_{16}(3)$ and the HOECHST nuclear dye. With a calibrated eyepiece reticle fluorescently labeled eosinophils in each lymph node cross-section were enumerated and results expressed as eosinophils per square millimeter. To identify $\mathrm{T}$ cell-rich regions of lymph nodes, after fluorescent photographs were taken, some sections were immunostained with anti-CD3 mAb (17A2; PharMingen, San Diego, California, USA) by a VECTASTAIN $\mathrm{ABC}$ immunoperoxidase kit with diaminobenzidine tetrahydrochloride substrate (Vector Laboratories, Burlingame, California, USA).

Flow cytometry. FITC-conjugated anti-I-A ${ }^{\mathrm{d}}$ (AMS32.1), anti-CD80 (16-10A1), and anti-CD86 (GL1) $\mathrm{mAbs}$ were from PharMingen. Eosinophils in PBS, 1 $\mathrm{mg} / \mathrm{mL} \mathrm{BSA}$, and $0.1 \mathrm{mg} / \mathrm{mL}$ sodium azide were stained at $4^{\circ} \mathrm{C}$ for 30 minutes with saturating quantities of $\mathrm{mAb}$ or matched FITC-conjugated isotypic IgG, washed in the same medium, and fixed in $0.5 \%$ paraformaldehyde. Flow cytometry was performed with a Becton Dickinson FACScan (Becton Dickinson Immunocytometry Systems, San Jose, California, USA). Antigen presentation to sensitized $T$ cells by eosinophils in vitro. To obtain antigen-sensitized T cells, 6 -week-old $\mathrm{BALB} / \mathrm{c}$ mice were immunized intraperitoneally with $10 \mu \mathrm{g}$ of antigen (OVA, BSA, or HGG) plus $1 \mathrm{mg}$ $\mathrm{Al}(\mathrm{OH})_{3}$. Spleens, taken 2 weeks later, were pressed through wire-mesh screens to separate cells. Erythrocytes were lysed with hypotonic saline. After passage over Sephadex G-10 columns to remove accessory cells, spleen cell suspensions were incubated with antiCD90-coated micromagnetic beads. By positive selection (Miltenyi Biotec MACS), greater than $97 \%$ pure T cells were obtained with viabilities greater than $98 \%$. Eosinophils were cultured with $5 \mathrm{ng} / \mathrm{mL}$ recombinant mouse GM-CSF (BioSource, Camarillo, California, USA) to sustain eosinophil viability during the 72hour lymphocyte proliferation assays. Antigen-sensitized T cells $\left(2 \times 10^{5}\right)$ were cultured in triplicate with designated numbers of antigen-elicited and -exposed airway-derived eosinophils in supplemented RPMI1640 in flat-bottomed 96-well plates. In some experiments, anti-CD80 (16-10A1; $10 \mu \mathrm{g} / \mathrm{mL})$ and/or antiCD86 (GL1; $10 \mu \mathrm{g} / \mathrm{mL}$ ) mAbs (PharMingen), or a soluble fusion protein of extracellular domain of cytotoxic T lymphocyte-associated antigen-4 and the Fc portion of IgG (CTLA-4Ig; $5 \mu \mathrm{g} / \mathrm{mL}$; Ancell Co., Bayport, Minnesota, USA) or control Igs (rat $\operatorname{IgG}_{2 a}, 10$ $\mu \mathrm{g} / \mathrm{mL}$, for anti-CD80 and anti-CD86, and mouse $\mathrm{IgG}_{2 \mathrm{a}}, 5 \mu \mathrm{g} / \mathrm{mL}$, for CTLA-4Ig) were added. Triplicate cultures for each condition were incubated for 3 days at $37^{\circ} \mathrm{C}, 5 \% \mathrm{CO}_{2}$. After adding $1 \mu \mathrm{Ci}$ of ${ }^{3} \mathrm{H}$-thymidine per well (NEN Life Science Products Inc., Boston,
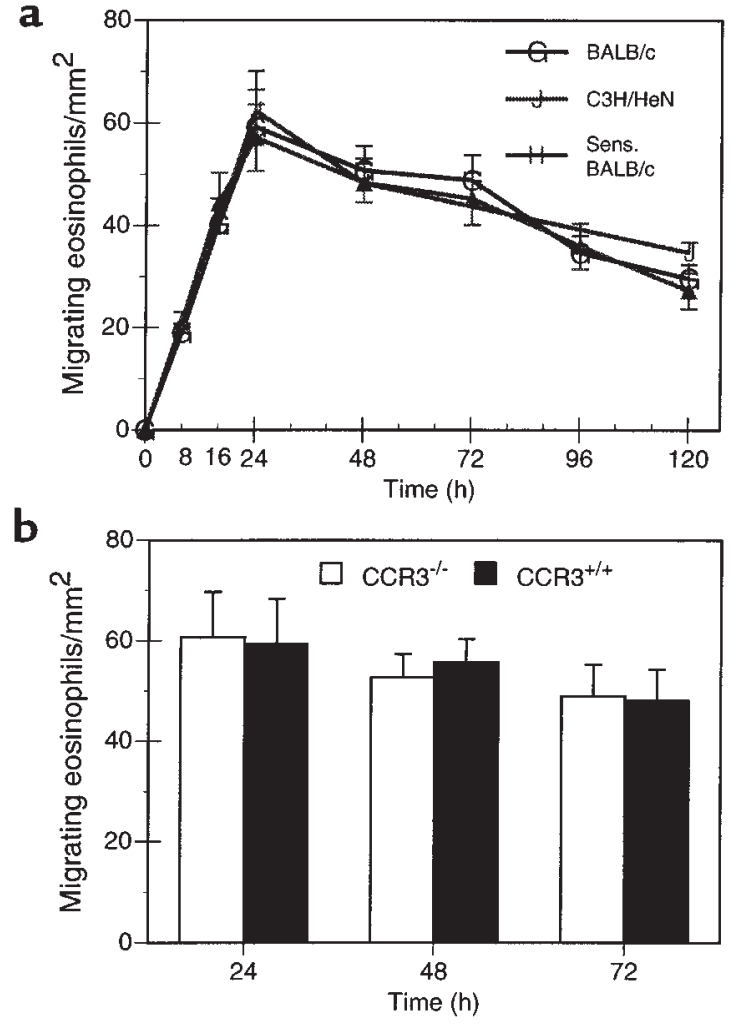

Figure 2

Eosinophil localization within paratracheal lymph nodes. After $\operatorname{DilC}_{16}(3)$-labeled eosinophils $\left(5 \times 10^{5}\right)$ were instilled into the tracheas of recipient mice, paratracheal lymph nodes were examined by fluorescence microscopy to enumerate migrated eosinophils per square millimeter, as described in Methods. (a) Kinetics of eosinophil appearance in lymph nodes for normal BALB/c mice receiving airway eosinophils isolated from OVA-sensitized and aerosol-challenged $\mathrm{BALB} / \mathrm{c}$ mice $(\mathrm{G})$, for normal $\mathrm{C} 3 \mathrm{H} / \mathrm{HeN}$ mice receiving peritoneal eosinophils isolated from IL-5 C3H/HeN transgenic mice $(\mathrm{J})$, and for OVA-sensitized BALB/c mice receiving airway eosinophils isolated from OVA-sensitized and aerosol-challenged BALB/c mice $(H)$. Each result represents the mean \pm SEM from 6 mice. (b) Comparisons of the lymph node migration of $\mathrm{CCR}^{-/-}$and $\mathrm{CCR} 3^{+/+}$airway-derived eosinophils isolated from OVA-sensitized and aerosol-challenged mice instilled intratracheally into normal BALB/c mice. Each result represents the mean \pm SEM from 3 mice per group.

Massachusetts, USA), lymphocyte ${ }^{3} \mathrm{H}$-thymidine incorporation was determined 16-18 hours later, using a cell harvester and liquid scintillation spectrometry. To calculate inhibition, basal unstimulated proliferation was subtracted and percentage inhibition was calculated relative to its $\mathrm{mAb}$ control; results are means \pm SEM from 3 independent experiments.

Antigen presentation to sensitized $T$ cells by eosinophils in vivo. Mice were immunized intraperitoneally with 10 $\mu \mathrm{g}$ of either OVA, BSA, or $\mathrm{HGG}$ plus $1 \mathrm{mg} \mathrm{Al}(\mathrm{OH})_{3}$. Two week later, designated numbers of purified OVA-, BSA-, or HGG-exposed eosinophils in $50 \mu \mathrm{L}$ PBS were introduced into the tracheas of lightly anesthetized mice. In some experiments, OVA-sensitized mice were injected intravenously with anti-CD80 (100 


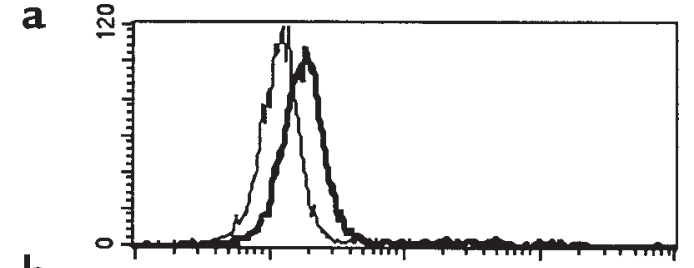

b

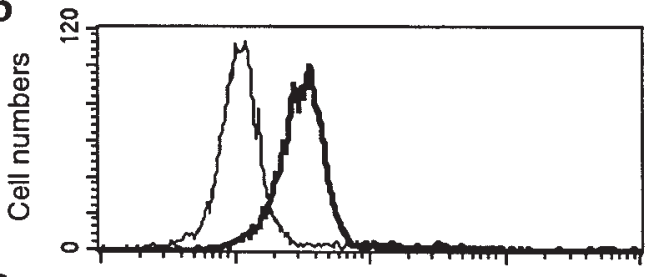

C

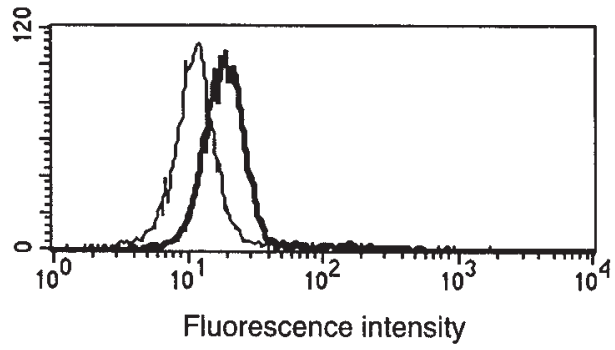

Figure 3

Expression of MHC class II molecules, CD80 and CD86, on airway eosinophils. Eosinophils, from BAL of BALB/c mice sensitized and aerosol-challenged with antigen, were stained with FITC-conjugated anti-I-A $(\mathbf{a})$, anti-CD80 (b), or anti-CD86 (c) mAbs (thick lines) and analyzed by flow cytometry in comparison with isotype control FITCconjugated IgG (thin lines). Results show a representative experiment from mice sensitized and challenged with OVA. Similar results were found with mice sensitized and aerosol challenged with BSA or HGG.

$\mu \mathrm{g})$, anti-CD86 $(100 \mu \mathrm{g})$, a combination of both antiCD80 and anti-CD86 mAbs (total of $200 \mu \mathrm{g}$ ), CTLA4Ig $(100 \mu \mathrm{g})$, or control Igs $(100 \mu \mathrm{g}$ each $)$ immediately before and 1 and 2 days after they received endotracheal instillations of $5 \times 10^{5}$ OVA-exposed eosinophils. At specified times thereafter, paratracheal lymph nodes were removed and teased into cell suspensions. Lymph node cells $\left(3 \times 10^{5}\right)$, cultured in triplicate, were pulsed with $1 \mu \mathrm{Ci}$ of ${ }^{3} \mathrm{H}$-thymidine per well and cultured for $16-18$ hours, with ${ }^{3} \mathrm{H}$-thymidine incorporation was determined as above.

Identity of $T$ cells responding in vivo to antigen-presenting eosinophils. OVA-, BSA-, or HGG-exposed eosinophils (5 $\times 10^{5}$ ) were instilled into the tracheas of either OVA-, BSA-, or HGG-immunized mice. Control mice received tracheal instillations of PBS or eosinophils rendered nonviable by $2 \%$ paraformaldehyde fixation. Three days later mice were injected twice intraperitoneally with 2 $\mathrm{mg}$ bromodeoxyuridine (BrdU; Sigma) in $0.2 \mathrm{~mL}$ sterile PBS at 30-minute intervals. Thirty minutes after the second injection, mice were sacrificed, and paratracheal lymph node $T$ cells were isolated and purified as above. After staining with phycoerythrin-conjugated (PE-conjugated) CD4 (L3T4) and CD8 (Ly-2) mAbs (PharMingen) and T cells (> 96\% pure) were washed, resuspended in cold $0.15 \mathrm{M} \mathrm{NaCl}$, and fixed with cold $95 \%$ ethanol. T cells were held for 30 minutes on ice, washed with PBS, fixed with PBS containing $0.5 \%$ paraformaldehyde and $0.01 \%$ Tween 20 for 1 hour, and incubated at $37^{\circ} \mathrm{C}$ with 50 Kunitz units DNase I (Sigma) in $0.15 \mathrm{M} \mathrm{NaCl}, 4.2 \mathrm{mM} \mathrm{MgCl} 2, \mathrm{pH} 5$, for 10 minutes. After washing, $\mathrm{T}$ cells were incubated with FITC-conjugated anti-BrdU mAb (B44; Becton Dickinson) for 30 minutes at room temperature and analyzed using a FACScan flow cytometer with Cellquest software (Becton Dickinson).

\section{Results}

Trafficking of airway eosinophils. To evaluate the capacity of eosinophils within the tracheobronchial lumen to enter tissues, eosinophils recovered from the airways of antigen-sensitized mice after aerosol challenges were fluorescently labeled ex vivo with $\operatorname{DiIC}_{16}(3)$ and instilled into the tracheas of both normal and antigensensitized mice. Fluorescently labeled eosinophils entered paratracheal lymph nodes within 8 hours (Figures 1 and 2). By 8 hours, labeled eosinophils were visible in the subcapsular region and streaming through the subcapsular sinus. With time the number of eosinophils entering the regional lymph nodes increased, peaking at 24 hours and persisting for at least 120 hours (Figure 2a). By 24 hours after instillation, labeled eosinophils were predominantly located in the T-cell regions of lymph nodes, with only occasional eosinophils in the B-cell areas or the subcapsular sinus (Figure 1). Within 120 hours, fluorescently labeled eosinophils were not detected by microscopy in sections of other examined extrapulmonary lymph nodes, or the spleen, or the liver.

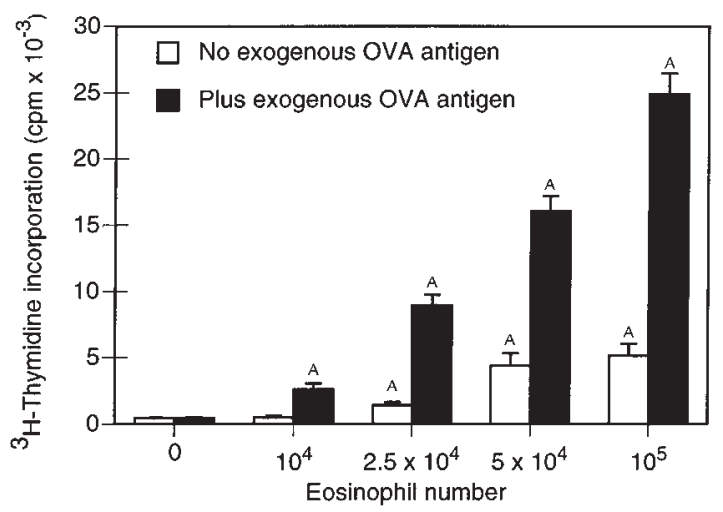

Figure 4

In vitro stimulation of sensitized T-lymphocyte proliferation by antigen-exposed and antigen-elicited airway eosinophils. OVA-sensitized T cells $\left(2 \times 10^{5}\right)$ were incubated with different numbers of airway eosinophils purified from OVA-sensitized and OVA-challenged mice in the absence and presence of $200 \mu \mathrm{g} / \mathrm{mL}$ exogenous OVA antigen. After 72 hours, cultures were pulsed with ${ }^{3} \mathrm{H}$-thymidine, and ${ }^{3} \mathrm{H}$ thymidine incorporation was determined 16-18 hours later. Results are mean ${ }^{3} \mathrm{H}$-thymidine incorporation $\pm \mathrm{SD}$ from triplicate cultures. Data from a single experiment are representative of similar findings from 3 other experiments. ${ }^{A} P<0.05$, in comparison with values for no eosinophils, by ANOVA. 

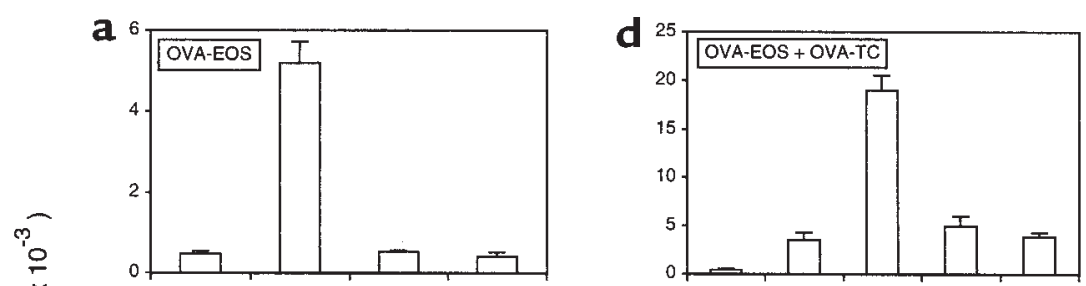

b 6

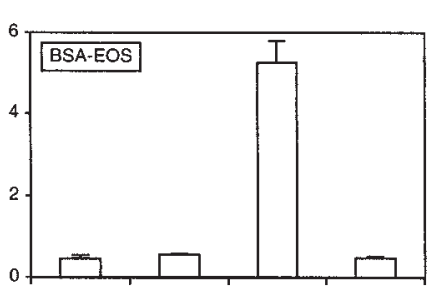

$c$

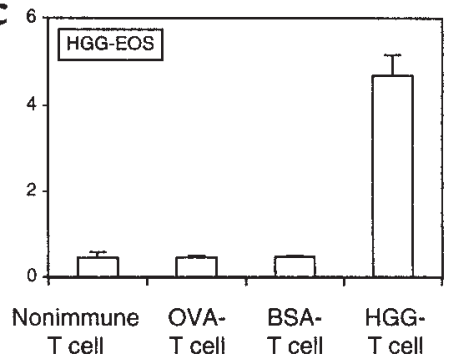

e 25

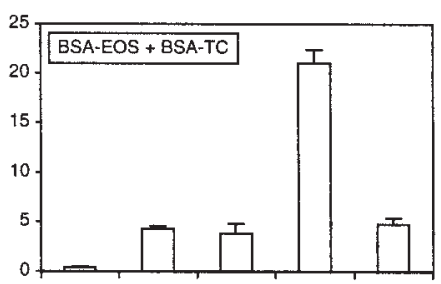

f

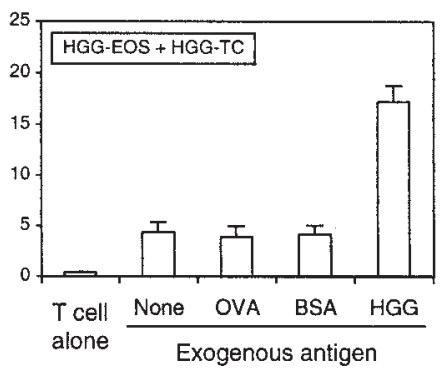

\section{Figure 5}

Antigen specificity of in vitro T-cell proliferation induced by antigen-exposed and antigen-elicited airway eosinophils. OVAexposed (a), BSA-exposed (b), and HGG-exposed (c) airway eosinophils (EOS) were cocultured with nonimmune or OVA-, BSA-, or HGG-sensitized T cells without exogenous antigen. OVA-sensitized (d), BSA-sensitized (e), and HGG-sensitized (f) $T$ cells (TC) were cocultured without eosinophils and exogenous antigen or with eosinophils purified from the airways of OVA-challenged (d), BSA-challenged (e), or HGG-challenged (f) mice with or with 200 $\mu \mathrm{g} / \mathrm{mL}$ of exogenous OVA, BSA, or HGG. After 72 hours, cultures of $5 \times 10^{4}$ eosinophils and $2 \times 10^{5} \mathrm{~T}$ cells were pulsed with ${ }^{3} \mathrm{H}$-thymidine, and ${ }^{3} \mathrm{H}$-thymidine incorporation was determined $16-18$ hours later. Results are mean ${ }^{3} \mathrm{H}$-thymidine incorporation \pm SD from triplicate cultures. The data from a single experiment are representative of similar findings from 3 other experiments.
The migration of eosinophils from the endotracheal lumen into regional lymph nodes was not limited to antigen-elicited airway-derived eosinophils. Peritoneal eosinophils purified from IL-5 C3H/HeN transgenic mice exhibited identical homing to paratracheal lymph nodes when instilled intratracheally into normal $\mathrm{C} 3 \mathrm{H} / \mathrm{HeN}$ mice (Figure 2a). Because homing of eosinophils within mucosal tissues may be governed by eotaxin expression (18), we assessed whether eosinophil migration from the airways to regional lymph nodes was directed by eotaxin expressed in recipient mice. The migration of eosinophils obtained following aerosol antigen challenge of mice with genetic deletion of the CCR3 receptor for eotaxin (and related chemokines) were examined. CCR $3^{-/-}$eosinophils exhibited identical homing to $C C R 3^{+/+}$eosinophils from wild-type mice (Figure 2b).

Expression of MHC class II, CD80, and CD86 molecules on airway eosinophils. Human blood eosinophils exhibit low or undetectable expression of class II MHC proteins (7), whereas class II MHC is present on airway eosinophils of asthmatic or after airway antigen challenge in atopic human subjects $(14,15,21)$. We determined if murine eosinophils from the airways of antigen-sensitized and -challenged mice expressed molecules involved in the presentation of exogenous antigens. Airway eosinophils recovered from antigen-sensitized and challenged BALB/c mice expressed high levels of I-A ${ }^{\mathrm{d}}$ (Figure 3a). In contrast, as reported previously (22), peritoneal eosinophils from IL-5 transgenic mice did not express class II MHC proteins (not shown) when isolated, although these eosinophils can be readily induced to express class II proteins in vitro (22). Airway eosinophils, like peritoneal eosinophils from IL-5 transgenic mice (22) (not shown), also expressed high levels of CD80 and CD86 (Figure 3, b and c), two B7 proteins with recognized roles as costimulatory signals for T-cell responses (23).

Eosinophil presentation of airway antigens to sensitized $T$ cells. We assessed the capacity of eosinophils recruited into the airways by aerosol antigen challenge to process and present antigen to which they were exposed within the airways. Splenic T cells from OVA-immunized mice were cultured with increasing numbers of eosinophils purified from the airways of mice challenged with aerosolized OVA (Figure 4). In the absence of eosinophils as APCs, T cells did not proliferate even when incubated with $200 \mu \mathrm{g} / \mathrm{mL}$ of exogenous antigen, confirming the absence of contaminating APCs among the purified $\mathrm{T}$ cells. The addition of eosinophils exposed to OVA in vivo yielded significant eosinophil dose-dependent increases in T-cell proliferation, indicative that eosinophils were presenting in vivo-derived and-processed OVA peptides to the sensitized T cells. The addition of exogenous antigen to these eosinophil-T cell cocultures yielded even greater eosinophil dose-dependent proliferation demonstrating that eosinophils were further processing in vitro-derived antigen and serving as APCs.

To ascertain that the heightened T-cell proliferation seen with eosinophils exposed in vivo to antigen was not due to a mitogenic or antigen-independent stimulatory process, the antigen specificity of the eosinophil APC function was evaluated. With OVA, BSA, and HGG as independent antigens to elicit antigen-specific $\mathrm{T}$ cells and to elicit airway eosinophils by aerosol 


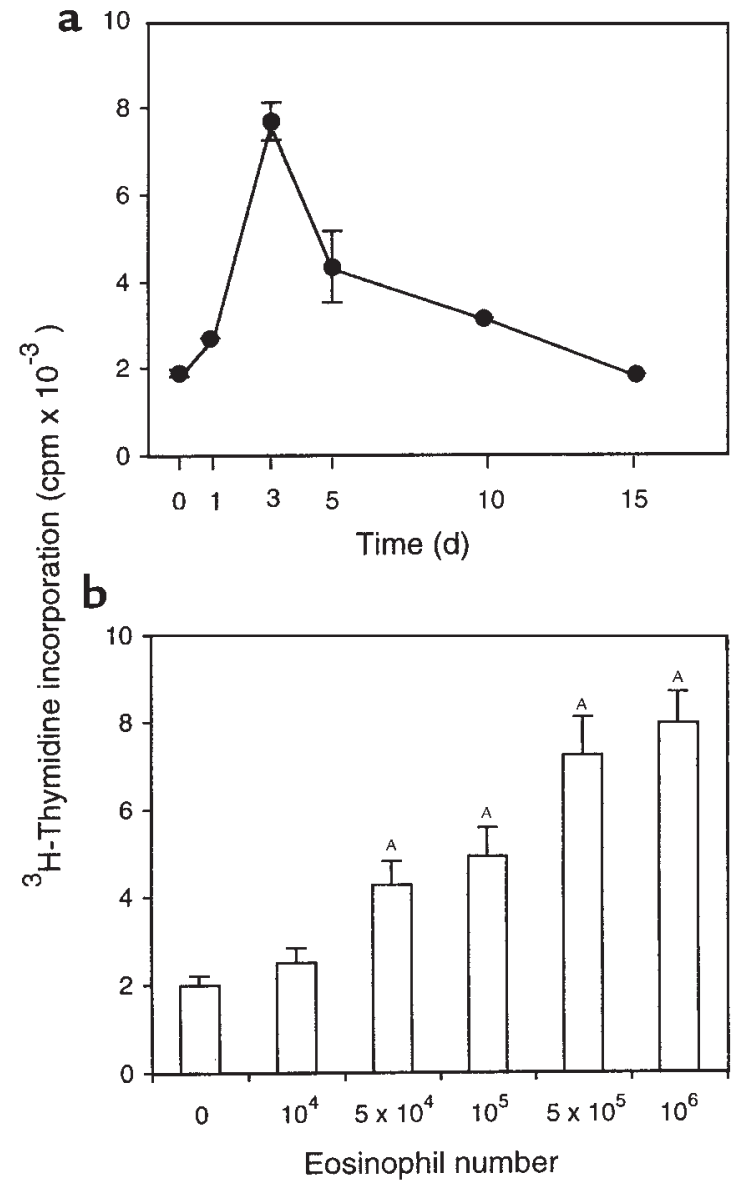

Figure 6

Kinetics and dose dependence of in vivo T-cell proliferation elicited by antigen-exposed and antigen-elicited airway eosinophils. (a) OVA-elicited airway eosinophils $\left(5 \times 10^{5}\right)$ were instilled into the tracheas of OVAsensitized mice, and paratracheal lymph nodes were taken at indicated times and evaluated for in vivo-induced proliferation. (b) Increasing numbers $\left(10^{4}-10^{6}\right)$ of OVA-elicited airway eosinophils were instilled into the tracheas of OVA-immunized mice, and paratracheal lymph nodes, taken 3 days after instillation, were evaluated for in vivo-induced proliferation. In each experiment $3 \times 10^{5} \mathrm{lymph}$ node cells were pulsed immediately with ${ }^{3} \mathrm{H}$-thymidine, incubated for 18 hours, and assessed for ${ }^{3} \mathrm{H}$-thymidine incorporation. Results are the mean ${ }^{3} \mathrm{H}$-thymidine incorporation \pm SEM of 3 independent experiments. ${ }^{A} P<0.01$, compared with controls in which no eosinophils were used, by ANOVA.

challenge, T-cell proliferative responses were found only when $T$ cells were cocultured with eosinophils exposed to the homologous antigen (Figure 5, a-c). That eosinophils were the only APCs present in these cultures with purified and antigen-specific $T$ cells was demonstrated by the failure of $\mathrm{T}$ cells to proliferate in response to exogenous antigens in the absence of eosinophils (Figure 5, d-f). Again, eosinophils exhibited antigen-specific APC function in response to exogenous antigen added during T-cell proliferation assays, as well as their capacity to process and present antigen to which they were exposed in vivo within the airways.

To evaluate whether eosinophils were providing requisite B7 costimulatory signals for their APC function (23), we assessed the roles of CD80 and CD86 as cos- timulatory signals in eosinophil antigen presentation in vitro to OVA-sensitized T cells. We cultured $2 \times 10^{5}$ OVA-sensitized $\mathrm{T}$ cells and $5 \times 10^{4}$ OVA-exposed eosinophils without exogenous OVA in the presence or absence of inhibitory concentrations of anti-CD80 $\mathrm{mAb}$, anti-CD86 mAb, a combination of both, or CTLA-4Ig. Anti-CD80 and anti-CD86 mAb each partially blocked proliferation, causing $43.9 \pm 2.8 \%$ and $51.4 \pm 4.6 \%$ inhibition (mean $\pm \mathrm{SEM}, n=3$ ), respectively. Both anti-CD80 and anti-CD86 blocking $\mathrm{mAbs}$ in combination, or CTLA-4Ig, yielded even greater inhibition of eosinophil-elicited T-cell proliferation (84.7 \pm $2.7 \%$ and $90.0 \pm 1.9 \%$, respectively).

Antigen presentation by airway eosinophils to primed $T$ cells in vivo. To test the APC function of antigen-exposed eosinophils in vivo different numbers of eosinophils from either OVA-, BSA-, or HGG-sensitized and -challenged mice were instilled into tracheas of either OVA-, BSA-, or HGG-sensitized mice. At specific times thereafter, paratracheal lymph nodes were taken and tested for in vivo proliferation by being pulsed immediately with ${ }^{3} \mathrm{H}$-thymidine and incubated for $16-18$ hours. In OVA-sensitized mice that received $5 \times 10^{5}$ OVAexposed eosinophils, in vivo T-cell proliferative responses in the paratracheal nodes developed within 1 day, peaked at day 3 , and then declined over 1 week (Figure 6a). The in vivo T-cell proliferative responses increased with increasing numbers of OVA-exposed eosinophils instilled into the tracheas (Figure 6b). Like the in vitro experiments, eosinophil-induced in vivo T-cell proliferation was also antigen specific, because eosinophils from OVA-sensitized and -challenged mice could only induce proliferation responses of $\mathrm{T}$ cells from OVA-sensitized mice, but not from BSA- or HGG-sensitized mice, and the same results were observed with BSA- or HGG-exposed airway eosinophils (Figure 7).

To delineate the lymph node T-cell subsets induced to proliferate in vivo by the instilled airway eosinophils from antigen-sensitized and aerosol-challenged mice, replicating $\mathrm{T}$ cells were labeled in vivo by BrdU incorporation. Lymph node $T$ cells were double-stained with FITC-conjugated anti-BrdU mAb and PE-conjugated anti-CD4 or anti-CD8 mAbs and analyzed by flow cytometry. Only low percentages of $\mathrm{BrdU}^{+} \mathrm{CD}^{+}$and $\mathrm{BrdU}^{+} \mathrm{CD} 8^{+}$cells were present in paratracheal lymph nodes of OVA-sensitized mice not given intratracheal eosinophil instillations (Figure 8, a and c). In contrast, the endotracheal instillation of OVA-exposed eosinophils led to significant increases in $\mathrm{BrdU}^{+} \mathrm{CD}^{+}$ (Figure $8 \mathrm{~b}$ ) but not in $\mathrm{BrdU}^{+} \mathrm{CD}^{+}$cells (Figure $8 \mathrm{~d}$ ). Percentages (mean \pm SEM) of $\mathrm{BrdU}^{+} \mathrm{CD}^{+}$cells in sensitized mice receiving eosinophil instillation $(7.7 \pm$ $0.5 \%)$ were much higher than that in control mice $(2.0$ $\pm 0.1 \% ; n=5 ; P=0.0003$, paired $t$ test). Similar results were found with BSA-immunized mice receiving BSAexposed eosinophils and HGG-immunized mice receiving HGG-exposed eosinophils (not shown). As expected, the endotracheal instillation of fixed, nonviable eosinophils induced no changes in percentages of 
either $\mathrm{BrdU}^{+} \mathrm{CD}^{+}$and $\mathrm{BrdU}^{+} \mathrm{CD}^{+}$cells (not shown).

Having established the APC functional activity of airway eosinophils in vivo, we then analyzed the role of B7 costimulatory molecules (23) in in vivo eosinophil antigen presentation to $\mathrm{T}$ cells by blocking CD80, CD86, or both. Treatment of OVA-sensitized mice with either anti-CD80 or anti-CD86 mAb each partially suppressed the peak day-3 T-cell proliferation elicited in vivo by tracheal instillation of OVA-exposed eosinophils $(42.0 \pm 1.7 \%$ and $54.4 \pm 6.2 \%$; mean inhibitions \pm SEM, $n=3$, respectively). Treatments with either the combination of anti-CD80 and anti-CD $86 \mathrm{mAbs}$ or CTLA-4Ig fusion protein to block both B7-1- and B72-mediated costimulation (23) each resulted in about $90 \%$ inhibition of in vivo eosinophil-elicited T-cell proliferation $(90.1 \pm 2.2 \%$ and $90.2 \pm 2.2 \%$, respectively; mean inhibitions \pm SEM, $n=3$ ).

\section{Discussion}

Although the presence of eosinophils in respiratory secretions is a hallmark of allergic airways diseases (24), little consideration has been given to the potential physiologic function of intraluminal eosinophils and whether eosinophils might migrate back across the epithelium to re-enter submucosal tissues. Like lemmings (animals that migrate in mass and mythically plunge into the sea to their death), it is conventionally assumed that eosinophil transit through the airway epithelium into the lumen is a terminal event. Our studies aimed at evaluating the capabilities of endobronchial eosinophils to enter host tissues and to function as APCs in response to antigens that these eosinophils encountered within the airways.

The instillation of fluorescently labeled eosinophils into the tracheal lumina of mice allowed the transit of these exogenous eosinophils to be monitored. Both eosinophils recovered from antigen-challenged airways, likely analogous to eosinophils in the airways of asthmatic humans, and peritoneal eosinophils from IL-5 transgenic mice exhibited identical homing to peritracheal lymph nodes (Figures1 and 2). Based on the numbers of eosinophils in examined $10-\mu \mathrm{m}$ thick sections of peritracheal lymph nodes and the measured mass of lymph nodes, approximately $10 \%$ of eosinophils instilled into the airways had become resident at 24 hours within the sampled peritracheal lymph nodes; this would not include other minor lymph nodes or bronchial-associated lymphoid sites that were not evaluated. Thus, even in normal mice whose airway epithelium is intact and not subject to potentially stimulatory inflammatory mediators, intraluminal eosinophils facilely migrated into regional lymph nodes.

Although intraluminal eosinophils entered peritracheal tissues and trafficked to lymph nodes, the route and mechanisms of this migration are not yet defined. Eosinophils can traffic in both directions across confluent intestinal epithelial monolayers (25). The production by airway epithelial cells of cytokines and chemokines active in eliciting eosinophil migration, such as IL-16
(16), RANTES $(17,19)$, and eotaxin (18), is usually considered as a means to direct eosinophil migration into the airway's lumen. Alternatively, these epitheliumexpressed chemoattractants might function to recruit some eosinophils from the lumen back toward the epithelium. RANTES is specifically released in a polarized fashion at the apical surface of airway epithelial cells, and this apical expression of RANTES is even more prominent in the airways of those with asthma (19). Hence, for this eosinophil chemotactic chemokine there would be a concentration gradient within the lumen

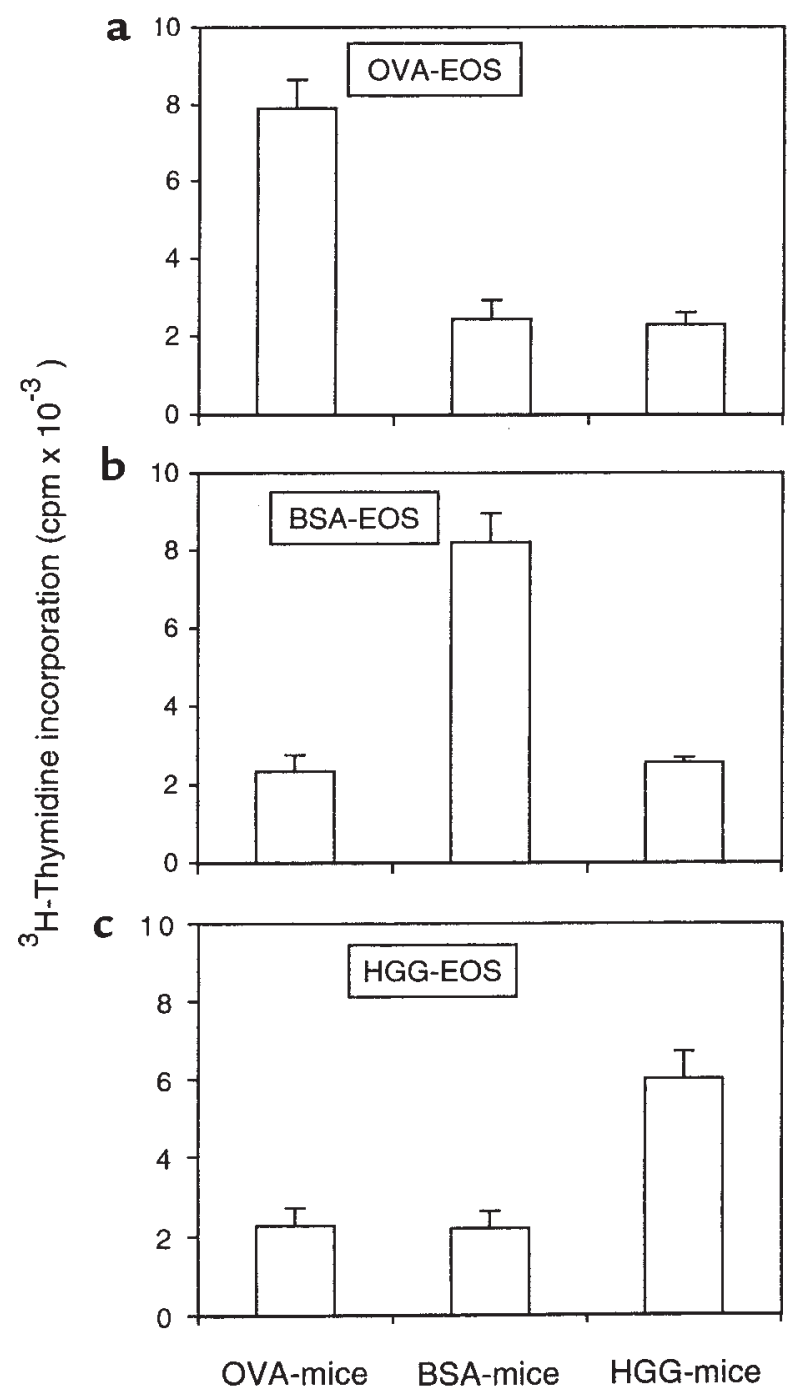

Figure 7

The antigen specificity of in vivo T-cell proliferation elicited by antigen-exposed and antigen-elicited airway eosinophils. OVA-elicited (a), BSA-elicited (b), and HGG-elicited (c) airway eosinophils $(5 \times$ $10^{5}$ ) were instilled into the tracheas of nonimmune or OVA-, BSA-, or HGG-immunized mice. Paratracheal lymph nodes, taken 3 days after instillation, were evaluated for in vivo-induced proliferation. Lymph node cells $\left(3 \times 10^{5}\right)$ were pulsed with ${ }^{3} \mathrm{H}$-thymidine, incubated for 16-18 hours, and assessed for ${ }^{3} \mathrm{H}$-thymidine incorporation. Results are the mean ${ }^{3} \mathrm{H}$-thymidine incorporation $\pm \mathrm{SD}$ of triplicate cultures. The data with a single experiment are representative of similar findings with 3 other experiments. 

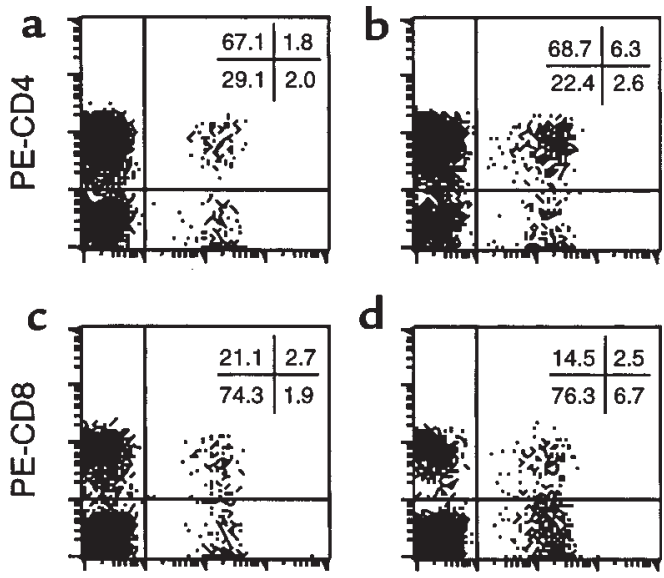

\section{Figure 8}

$\mathrm{CD} 4^{+}$but not $\mathrm{CD} 8^{+} \mathrm{T}$ cells respond in vivo to antigen-presenting eosinophils. PBS $(\mathbf{a}, \mathbf{c})$ or antigen-exposed eosinophils $(\mathbf{b}, \mathbf{d})$ were instilled into the tracheas of antigen-sensitized mice. As described in Methods, mice were administered BrdU, and T cells were purified from lymph nodes 3 days after eosinophil instillation, double-stained with FITC-anti-BrdU mAb and PE-anti-CD4 (a, b) or anti-CD8 mAb (c, d) and analyzed by flow cytometry. Results show 1 experiment representative of 5 independent experiments from OVA-immunized mice receiving OVA-exposed eosinophils; similar results were also found in BSA-immunized mice receiving BSA-exposed eosinophils and HGG-immunized mice receiving HGG-exposed eosinophils. Numbers specify the percentage of cells in each of the 4 quadrants.

increasing toward the apical surface of the epithelium, which could direct eosinophil migration from the lumen.

A candidate mechanism whereby endobronchial eosinophils home to regional lymph nodes might involve eosinophil chemoattraction by eotaxin or other chemokines. Eotaxin is expressed by the airway's epithelium and may be generated in lymph nodes (18). This mechanism based on chemokines, such as eotaxin, that signal eosinophils through the CCR3 receptor, however, is not likely to be involved. Eosinophils lacking CCR3 homed identically to peritracheal lymph nodes (Figure 2). Whether other chemokine attractants or eosinophil-expressed integrins, such as $\alpha 4 \beta 7$, may be involved in the localization of eosinophils to peritracheal lymph nodes remains to be established.

If eosinophils within the airways are to function in presenting inhaled antigens to $T$ cells, homing to lymph nodes would be an important necessary attribute of eosinophils, but by itself would not be sufficient. Eosinophils would need to express class II MHC proteins, and likely costimulatory molecules, and exhibit a capacity to effectively internalize, process, and present airway-derived antigens to $\mathrm{T}$ cells. Our studies of murine eosinophils recovered from the airways after inhalational antigen challenges confirmed that these eosinophils exhibited these requisite features. Murine eosinophils from the antigen-challenged airways of sensitized BALB/c mice, like human airways eosinophils $(14,15,21)$, expressed class II MHC proteins (Figure 3).
Murine eosinophils also expressed CD80 and CD86 (Figure 3) (22), again comparable to human eosinophils that express at least CD86 (6). In mice, the inhalational exposure of airway eosinophils to any of 3 antigens was shown to be sufficient for these airway eosinophils, by antigen-specific and CD80- and CD86-dependent means, to present antigens to $\mathrm{T}$ cells in in vitro assays (Figures 4 and 5). These findings using in vivo exposures of eosinophils to antigens solely within the airways extend previous studies, demonstrating that murine $(10,22)$ and human $(9,21,26,27)$ eosinophils exposed to antigens only in vitro may serve as APCs.

Many cell types have the capacity to serve as APCs. Thus, for eosinophils, a critical test was to ascertain whether eosinophils within the airways, after encountering inhaled antigens, could function to present antigens and elicit T-cell responses in vivo. Our studies established not only that endotracheal eosinophils homed to $\mathrm{T}$ cell-rich regions of peritracheal lymph nodes (Figure 1), but also that these antigen-exposed, airway-derived eosinophils, when instilled into the airways of antigen-sensitized mice, were capable of stimulating T-cell proliferative responses in vivo within peritracheal lymph nodes (Figure 6). These eosinophil APC-mediated, in vivo T-cell proliferative responses were antigen specific (Figure 7), CD80/CD86 dependent (Figure 8), and limited to $\mathrm{CD}^{+} \mathrm{T}$ cells.

Compared with other cell types that are APCs, several properties of eosinophils likely combine to endow them with distinct roles as APCs. First, the normal localization of eosinophils within mucosal tissues of the respiratory, gastrointestinal, and lower genitourinary tracts (28) would position them to encounter foreign antigens at these mucosal surfaces. Moreover, in allergic airway diseases, eosinophils are characteristically found directly within the lumen and airway secretions $(11,12)$ and could directly interact with inhaled allergens within the airways. Second, eosinophils, as demonstrated in this study, have the capacity to migrate from the lumen into regional lymph nodes. Both alveolar macrophages (29) and dendritic cells $(30,31)$ can also migrate from the airways into tissues, but in vivo antigen-pulsed macrophages do not transfer processed peptides to dendritic cells (32) and alveolar macrophages do not function as APCs $(33,34)$.

A third feature of eosinophils that might be important in their role as APCs at airway surfaces is their capacity to interact with particulate antigens. Whereas dendritic cells and B cells effectively present soluble protein antigens, they are unable to handle particulate antigens (35). In the respiratory tract inhaled allergens are particulate (36). Thus, dendritic cells may be ill suited to process inhaled particulate aeroallergens. The principal cells recognized to ingest particulate antigens are phagocytic macrophages (37), but alveolar macrophages are not effective APCs and even antagonize APC function of dendritic cells $(33,34,38)$. Alternatively, eosinophils would be well suited to han- 
dle particulate antigens because eosinophils are phagocytic, characteristically engage large, even nonphagocytosable, multicellular targets, and accumulate early at tissue sites of particulate antigens (39). Finally, the expression of IgG, IgE, and IgA immunoglobulin receptors on eosinophils might enable immunoglobulin-facilitated enhancement of antigen uptake and presentation by eosinophils, as shown for other APCs $(40,41)$. In mucosal sites eosinophils will be exposed to antibodies of all 3 classes, including IgG, $\operatorname{IgE}$, and IgA antibodies to inhaled allergens.

In conclusion, this study has shown that when instilled in the airways of sensitized recipient mice, eosinophils exposed to inhaled antigens within the airways of donor mice, migrate from the airways and home to regional nodes where they serve as antigen-specific, CD80- and CD86-dependent APCs to elicit the proliferation of nodal $\mathrm{CD} 4^{+} \mathrm{T}$ cells. In allergic airway diseases, where class II MHC-expressing eosinophils are present in the lumina, eosinophil trafficking and functioning as APCs may contribute to or modulate the nature of local CD4 ${ }^{+} \mathrm{T}$-cell responses to inhaled allergens.

\section{Acknowledgments}

This work was supported by NIH grants AI-20241, AI22571, HL-46563, and AI-41995. We appreciate the assistance of Alain Sauty in initiating these studies and thank Akira Tominaga for providing IL-5 transgenic mice. H.Z. Shi was a postdoctoral fellow from Department of Internal Medicine, First Affiliated Hospital, Guangxi Medical University, People's Republic of China.

1. Seminario, M.C., and Gleich, G.J. 1994. The role of eosinophils in the pathogenesis of asthma. Curr. Opin. Immunol. 6:860-864.

2. Foster, P.S., Hogan, S.P., Ramsay, A.J., Matthaei, K.I., and Young, I.G. 1996. Interleukin 5 deficiency abolishes eosinophilia, airways hyperreactivity, and lung damage in a mouse asthma model. J. Exp. Med. 183:195-201.

3. Iwamoto, I., Nakajima, H., Endo, H., and Yoshida, S. 1993. Interferon gamma regulates antigen-induced eosinophil recruitment into the mouse airways by inhibiting the infiltration of $\mathrm{CD}^{+}{ }^{+} \mathrm{T}$ cells. J. Exp. Med. 177:573-576.

4. Bentley, A.M., et al. 1993. Increases in activated T lymphocytes, eosinophils, and cytokine mRNA expression for interleukin-5 and granulocyte/macrophage colony-stimulating factor in bronchial biopsies after allergen challenge in atopic asthmatics. Am. J. Respir. Cell Mol. Biol. 8:35-42.

5. Ohkawara, Y., et al. 1996. CD40 expression by human peripheral blood eosinophils. J. Clin. Invest. 97:1761-1766.

6. Woerly, G., et al. 1999. Expression of CD28 and CD86 by human eosinophils and role in the secretion of type 1 cytokines (interleukin 2 and interferon $\gamma$ ): inhibition by immunoglobulin A complexes. J. Exp. Med. 190:487-495.

7. Lucey, D.R., Nicholson-Weller, A., and Weller, P.F. 1989. Mature human eosinophils have the capacity to express HLA-DR. Proc. Natl. Acad. Sci. USA. 86:1348-1351.

8. Lacy, P., and Moqbel, R. 1997. Eokines: synthesis, storage and release from human eosinophils. Mem. Inst. Oswaldo Cruz. 92(Suppl.):H125-H133.

9. Weller, P.F., et al. 1993. Accessory cell function of human eosinophils: HLA-DR dependent, MHC-restricted antigen-presentation and interleukin-1a formation. J. Immunol. 150:2554-2562.

10. Del Pozo, V., et al. 1992. Eosinophil as antigen-presenting cell: activation of $\mathrm{T}$ cell clones and $\mathrm{T}$ cell hybridoma by eosinophils after antigen processing. Eur. J. Immunol. 22:1919-1925.

11. Pin, I., et al. 1992. Changes in the cellular profile of induced sputum after allergen-induced asthmatic responses. Am. Rev. Respir. Dis. 145:1265-1269.

12. Foresi, A., et al. 1997. Eosinophils, mast cells, and basophils in induced sputum from patients with seasonal allergic rhinitis and perennial asth- ma: relationship to methacholine responsiveness. J. Allergy Clin. Immunol. 100:58-64.

13. Hansel, T.T., et al. 1991. Sputum eosinophils from asthmatics express ICAM-1 and HLA-DR. Clin. Exp. Immunol. 86:271-277.

14. Sedgwick, J.B., et al. 1992. Comparison of airway and blood eosinophil function after in vivo antigen challenge. J. Immunol. 149:3710-3718.

15. Mengelers, H.J., et al. 1994. Immunophenotyping of eosinophils recovered from blood and BAL of allergic asthmatics. Am. J. Respir. Crit. Care Med. 149:345-351.

16. Laberge, S., et al. 1997. Increased expression of interleukin-16 in bronchial mucosa of subjects with atopic asthma. Am. J. Respir. Cell Mol. Biol. 17:193-202.

17. Stellato, C., et al. 1995. Expression of the chemokine RANTES by a human bronchial epithelial cell line. Modulation by cytokines and glucocorticoids. J. Immunol. 155:410-418.

18. Rothenberg, M.E. 1999. Eotaxin. An essential mediator of eosinophil trafficking into mucosal tissues. Am. J. Respir. Cell Mol. Biol. 21:291-295.

19. Taguchi, M., et al. 1998. Patterns for RANTES secretion and intercellular adhesion molecule 1 expression mediate transepithelial $T$ cell traffic based on analyses in vitro and in vivo. J. Exp. Med. 187:1927-1940.

20. Tominaga, A., et al. 1991. Transgenic mice expressing a B cell growth and differentiation factor gene (interleukin 5) develop eosinophilia and autoantibody production. J. Exp. Med. 173:429-437.

21. Hansel, T.T., et al. 1992. Induction and function of eosinophil intercellular adhesion molecule-1 and HLA-DR. J. Immunol. 149:2130-2136.

22. Tamura, N., et al. 1996. Requirement of CD80 and CD86 molecules for antigen presentation by eosinophils. Scand. J. Immunol. 44:229-238.

23. McAdam, A.J., Schweitzer, A.N., and Sharpe, A.H. 1998. The role of B7 co-stimulation in activation and differentiation of $\mathrm{CD} 4^{+}$and $\mathrm{CD}^{+} \mathrm{T}$ cells. Immunol. Rev. 165:231-247.

24. Persson, C.G. 1997. Centennial notions of asthma as an eosinophilic, desquamative, exudative, and steroid-sensitive disease. Lancet. 350:1021-1024.

25. Resnick, M.B., et al. 1995. Human eosinophils migrate across an intestinal epithelium in response to platelet-activating factor. Gastroenterology. 108:409-416.

26. Mawhorter, S.D., Kazura, J.W., and Boom, W.H. 1994. Human eosinophils as antigen-presenting cells: relative efficiency for superantigen- and antigen-induced $\mathrm{CD} 4^{+} \mathrm{T}$-cell proliferation. Immunology. 81:584-591.

27. Wyss-Coray, T., et al. 1993. Antigen-presenting human T cells and antigen-presenting B cells induce a similar cytokine profile in specific $\mathrm{T}$ cell clones. Eur. J. Immunol. 23:3350-3357.

28. Rytömma, T. 1960. Organ distribution and histochemical properties of eosinophil granulocytes in the rat. Acta Pathol. Microbiol. Scand. 50(Suppl.):1-118.

29. Harmsen, A.G., Muggenburg, B.A., Snipes, M.B., and Bice, D.E. 1985. The role of macrophages in particle translocation from lungs to lymph nodes. Science. 230:1277-1280.

30. Havenith, C.E., van Miert, P.P., Breedijk, A.J., Beelen, R.H., and Hoefsmit, E.C. 1993. Migration of dendritic cells into the draining lymph nodes of the lung after intratracheal instillation. Am. J. Respir. Cell Mol. Biol. 9:484-488.

31. Xia, W., Pinto, C.E., and Kradin, R.L. 1995. The antigen-presenting activities of Ia+ dendritic cells shift dynamically from lung to lymph node after an airway challenge with soluble antigen. J. Exp. Med. 181:1275-1283

32. Crowley, M., Inaba, K., and Steinman, R.M. 1990. Dendritic cells are the principal cells in mouse spleen bearing immunogenic fragments of foreign proteins. J. Exp. Med. 383-386.

33. MacLean, J.A., et al. 1996. Sequestration of inhaled particulate antigens by lung phagocytes. A mechanism for the effective inhibition of pulmonary cell-mediated immunity. Am. J. Pathol. 148:657-666.

34. Holt, P.G., et al. 1993. Downregulation of the antigen presenting cell function(s) of pulmonary dendritic cells in vivo by resident alveolar macrophages. J. Exp. Med. 177:397-407.

35. van Rooijen, N. 1990. Antigen processing and presentation in vivo: the microenvironment as a crucial factor. Immunol. Today. 11:436-439.

36. Platt-Mills, T.A.E. 1992. Mechanisms of bronchial reactivity: the role of immunoglobulin E. Am. Rev. Respir. Dis. 145:S44-S47.

37. van Rooijen, N. 1992. Macrophages as accessory cells in the in vivo humoral immune response: from processing of particulate antigens to regulation by suppression. Semin. Immunol. 4:237-245.

38. Chelen, C.J., et al. 1995. Human alveolar macrophages present antigen ineffectively due to defective expression of B7 costimulatory cell surface molecules. J. Clin. Invest. 95:1415-1421.

39. Weller, P.F. 1991. The immunobiology of eosinophils. N. Engl. J. Med. 324:1110-1118

40. Maurer, D., et al. 1995. The high affinity IgE receptor (Fc epsilon RI) mediates IgE-dependent allergen presentation. J. Immunol. 154:6285-6290.

41. Gosselin, E.J., et al. 1992. Enhanced antigen presentation using human Fc gamma receptor (monocyte/macrophage)-specific immunogens. $J$. Immunol. 149:3477-3481. 